\title{
Preventing Metachronous Gastric Cancer after the Endoscopic Resection of Gastric Epithelial Neoplasia: Roles of Helicobacter pylori Eradication and Aspirin
}

\author{
Jiro Watari, Toshihiko Tomita, Katsuyuki Tozawa, Tadayuki Oshima, Hirokazu Fukui, and Hiroto Miwa \\ Division of Gastroenterology, Department of Internal Medicine, Hyogo College of Medicine, Nishinomiya, Japan
}

Whether Helicobacter pylori eradication actually reduces the risk of metachronous gastric cancer (MGC) development remains a controversial question. In this review, we addressed this topic by reviewing the results of clinical investigations and molecular pathological analyses of the roles of $\mathrm{H}$. pylori eradication and aspirin administration in the prevention of MGC. In regard to the clinical studies, the results of metaanalyses and randomized control trials differ from those of retrospective studies: the former trials show that $H$. pylori eradication has a preventive effect on MGC, while the latter studies do not. This discrepancy may be at least partly attributable to differences in the follow-up periods: $H$. pylori eradication is more likely to prevent MGC over a long-term followup period ( $\geq 5$ years) than over a short-term follow-up period. In addition, many studies have shown that aspirin may have an additive effect on MGC-risk reduction after $H$. pylori eradication has been achieved. Both $H$. pylori eradication and aspirin use induce molecular alterations in the atrophic gastritis mucosa but not in the intestinal metaplasia. Unfortunately, the molecular pathological analyses of these interventions have been limited by short follow-up periods. Therefore, a long-term prospective cohort is needed to clarify the changes in molecular events caused by these interventions. (Gut Liver 2020;14:281-290)

Key Words: Helicobacter pylori; Stomach neoplasms; Eradication; Aspirin; Pathology, molecular

\section{INTRODUCTION}

Helicobacter pylori infection is a major risk factor for the development of gastric cancer (GC). Infection by this bacterium is well known to be associated with the development of atrophic gastritis (AG), intestinal metaplasia (IM), dysplasia, and GC.' In Japan, therefore, the national insurance system has covered eradication therapy in $H$. pylori-infected chronic gastritis patients since 2013 to prevent the development of GC. ${ }^{2}$ Similarly, in 2014 the International Agency for Research on Cancer Working Group Report recommended that all countries explore the possibility of introducing population-based $H$. pylori screening and treatment programs as a strategy for GC prevention. ${ }^{3}$ To date, both meta-analyses ${ }^{4-10}$ and randomized controlled trials (RCTs) ${ }^{11,12}$ have found that $H$. pylori eradication appears to reduce the risk of GC. However, some retrospective studies ${ }^{13,14}$ and a prospective open-label trial ${ }^{15}$ have shown that $H$. pylori eradication did not reduce the incidence of metachronous GC (MGC) in patients who underwent endoscopic resection (ER). Thus, whether $H$. pylori eradication truly suppresses the development of MGC after ER remains controversial.

In addition, to reduce the risk of MGC after $H$. pylori eradication, additional combination treatments are needed, including treatments with anti-inflammatory agents. Many studies, including meta-analyses, have reported that aspirin and other nonsteroidal anti-inflammatory drugs (NSAIDs) reduced the risk of GC. ${ }^{16-22}$ The anti-carcinogenetic effects of these agents have been shown to contribute to the inhibition of the cyclooxygenase pathway. ${ }^{23,24}$

GC develops through the accumulation of genetic and epigenetic events. It has been widely reported that many of the molecular alterations of tumor-suppressor and tumor-related genes involve the development or progression of precancerous conditions, that is, atrophic mucosa (atrophy) and IM, as well as $\mathrm{GC}^{25-50}$

In this article, we review the effects of $H$. pylori eradication and aspirin/NSAIDs in preventing MGC along with the changes in molecular events induced by these interventions. Addition-

Correspondence to: Jiro Watari

Division of Gastroenterology, Department of Internal Medicine, Hyogo College of Medicine, 1-1, Mukogawa-cho, Nishinomiya 663-8501, Japan

Tel: +81-798-45-6662, Fax: +81-798-45-6661, E-mail: watarij@hyo-med.ac.jp

Received on March 4, 2019. Revised on May 11, 2019. Accepted on June 6, 2019. Published online September $25,2019$.

pISSN 1976-2283 eISSN 2005-1212 https://doi.org/10.5009/gnl19079

(a) This is an Open Access article distributed under the terms of the Creative Commons Attribution Non-Commercial License (http://creativecommons.org/licenses/by-nc/4.0) which permits unrestricted non-commercial use, distribution, and reproduction in any medium, provided the original work is properly cited. 
ally, we discuss efficient preventive strategies for MGC based on the clinical and basic literature, including original contributions, systematic reviews, and meta-analyses.

\section{PRECANCEROUS CONDITIONS OF THE GASTRIC MU- COSA AFTER ER OF GASTRIC EPITHELIAL NEOPLASIA}

Several studies have shown that precancerous conditions (i.e., AG and IM), are indicators of an increased risk for GC as compared with chronic gastritis in the absence of these lesions. ${ }^{51-53}$ Indeed, an international guideline known as the management of precancerous conditions and lesions of the stomach (MAPS) guideline considers AG and IM to be precancerous conditions and proposes the management for patients with those conditions. ${ }^{54}$ A prospective study by Uemura et al. ${ }^{55}$ reported that the relative risk (RR) for GC was 1.7 (95\% confidence interval [CI], 0.8 to 3.7) in moderate AG and 4.9 (95\% CI, 2.8 to 19.2) in severe AG compared to no AG or mild AG (control). It has recently been reported that $H$. pylori-associated GC is related to the extent of AG and IM based on the operative link on gastric atrophy (OLGA) and operative link on gastric IM (OLGIM) staging systems. ${ }^{56,57}$ The OLGA staging system may provide clinicians an immediate overall perception of GC risk, not only for intestinal-type GC but also for diffuse-type $\mathrm{GC}^{58}$ In this system, stages III and IV are significantly associated with GC development, ${ }^{59,60}$ which is consistent with the biological assumption that the extent and location of atrophy and IM correlate with the risk of GC. A recent meta-analysis also showed a significant association between OLGA/OLGIM stages III/IV and GC in prospective case-control studies (odds ratio [OR], 2.64; 95\% CI, 1.84 to 3.79; $\mathrm{p}<0.00001$ for the OLGA system and OR, 3.99; 95\% CI, 3.05 to $5.21 ; \mathrm{p}<0.00001$ for the OLGIM system). ${ }^{61}$ Meanwhile, the association between the higher stages (stage III/IV) of gastritis as defined by OLGA, but not OLGIM, and the risk of GC was significant in cohort studies. Since the OLGA staging system was reported to show lower interobserver agreement compared to the OLGIM system, a combination of the OLGA and OLGIM systems should be developed to more accurately assess the risk of GC in routine clinical practice. ${ }^{61}$ Patients with histologic IM or severe endoscopic atrophy were reported to be at increased risk of GC development even after $H$. pylori eradication; ${ }^{62}$ the cumulative 5-year incidences of GC were 3.2\% overall; 1.5\% (no IM), 5.3\% (IM in the antrum only), and 9.8\% (IM in the corpus); and 0.7\% (none/mild), 1.9\% (moderate), and 10\% (severe) based on the endoscopic atrophy grades according to the Kimura-Takemoto classification. ${ }^{63}$ Also, a recent meta-analysis showed that $H$. pylori eradication cannot reduce the risk of GC in patients with IM. ${ }^{5}$ In contrast, recent prospective studies with long-term follow-up showed the reversibility of AG and IM by H. pylori treatment. ${ }^{12,64} \mathrm{AG}$ was improved in the antrum and corpus beginning at 1-year of follow-up, and IM was improved in the antrum beginning at 5 or more years of follow-up and in the corpus beginning at 3 years of follow-up. ${ }^{64}$ Thus, this result indicates that it takes more time to reverse IM than AG. In addition, Choi et al. ${ }^{12}$ observed histologic improvement of the atrophy (48.8\%) and IM (36.6\%) at the corpus lesser curvature over a median follow-up period of 5.9 years. Clearly, it takes a longtime to substantially improve these precancerous conditions In addition, these findings indicate that the younger the age of eradication is, the better the prevention of precancerous conditions and ultimately GC will be. More recently, however, two intriguing studies reported that $H$. pylori eradication is effective for the prevention of GC at all ages, even in older individuals aged 60 years or older ${ }^{65}$ or below 70 years. ${ }^{66}$ Therefore, if $H$. pylori were eradicated even in populations of older individuals, the number of patients who have reached a "point of no return"67 might decrease from a long-term perspective. Asaka et al. ${ }^{68}$ concluded that the treatment would be expected to achieve nearly $100 \%$ prevention of GC in persons aged $<50$ years. A recent study by Leung et al. ${ }^{65}$ also concluded that because of the long lag time between $H$. pylori therapy and the prevention of GC development, $H$. pylori should be eradicated earlier to maximize the potential benefits of the treatment. Nonetheless, a prospective cohort with a large number of samples is needed to clarify the effect of $H$. pylori treatment for GC prevention in elderly populations.

\section{ROLE OF H. pylori ERADICATION IN REDUCING THE RISK OF MGC DEVELOPMENT}

H. pylori eradication significantly reduces the prevalence of primary GC by approximately one-third, as many investigators have mentioned. ${ }^{69}$ Some of the possible reasons for the reduction of GC by $H$. pylori eradication include: (1) eradication therapy inhibits new occurrence of GC; (2) eradication regresses or inhibits the growth of GC; and (3) eradication interferes with the discovery of GC. ${ }^{70}$ Therefore, when discussing reports on the reduction of GC risk by $H$. pylori eradication, the abovementioned possible mechanisms should be considered.

\section{Review of RCTs}

In 2008, the Japan Gast Study Group concluded in a multicenter, open-label RCT that $H$. pylori eradication after ER of GC significantly reduced MGC at 3-year follow-up; the hazard ratio (HR) for MGC was 0.339 (95\% CI, 0.157 to 0.729; $p=0.003){ }^{1}$ Thereafter, although Choi et al. ${ }^{15}$ performed a similar RCT in South Korea (median follow-up: 3 years), they found that eradication of $H$. pylori had no significant impact on the incidence of MGC. In any RCT, the follow-up period may affect the results; thus, a prolonged follow-up period of at least 5 years is needed to clarify the effects of $H$. pylori treatment. ${ }^{10}$ With regard to primary GC development after $H$. pylori eradication, the reduction of GC after eradication was significantly greater $(\mathrm{p}=0.01)$ in the groups with long-term ( $\geq 5$ years) follow-up (OR, 0.32; 
95\% CI, 0.24 to 0.43$)$ as compared to those with shorter followup (<5 years) (OR, 0.55; 95\% CI, 0.41 to 0.72$).{ }^{10}$ Interestingly, Choi et al. ${ }^{71}$ changed their previous negative conclusion after a long-term follow-up (median follow-up: 71.6 months) and ultimately found that $H$. pylori eradication significantly reduced the incidence of MGC. A recent RCT also showed that early GC patients who received $H$. pylori treatment had lower rates of MGC during a median follow-up of 5.9 years (HR, 0.50; 95\% CI, 0.26 to $0.94 ; \mathrm{p}=0.03){ }^{12}$ Taken together, these results suggest that $H$. pylori eradication may reduce MGC development in patients who are followed-up for a long term (at least $\geq 5$ years), but may not show a reduction in MGC development over a short-term follow-up ( $<5$ years).

\section{Review of meta-analyses}

To date, most meta-analyses have demonstrated that $H$. pylori eradication reduces the risk of MGC (Table 1). ${ }^{4-10}$ The preventive effects of $H$. pylori eradication are considered to be influenced by the following factors: (1) whether the MGC includes dysplasia and GC; (2) whether the lesions that underwent ER include not only GC but also dysplasia; (3) whether H. pylori-eradicated and naturally eradicated patients are treated identically as $H$. pylori-negative cases; (4) the study design; (5) the methodological quality of enrolled studies; and others. ${ }^{9}$ Even taking these factors into consideration, an effect was clearly detected, and its size was similar among these meta-analyses (RR, 0.39 to 0.52). Two meta-analyses reported that the magnitude of the protective effect induced by eradication is greater among individuals with a higher baseline GC risk, such as patients who have undergone ER of early GC, compared to primary GC patients. ${ }^{4,5}$ In contrast, a recent analysis by Sugano ${ }^{10}$ showed that the effect of the reduction induced by $H$. pylori eradication was higher in patients without GC at baseline (OR, 0.41; 95\% CI, 0.32 to 0.52) than in those with GC at baseline (OR, $0.51 ; 95 \% \mathrm{CI}, 0.40$ to 0.64 ) (Table 1). It may be difficult to clearly explain the reason for this discrepancy. Commonly, in patients with neoplastic lesions including AG, IM, and dysplasia, the effect of eradication on
GC development is decreased. ${ }^{67}$ In addition, GC is related to the extent of AG and IM according to the OLGA and OLGIM staging systems; ${ }^{56,57}$ thus, it is possible that the benefit of $H$. pylori eradication may be lower in patients with precancerous conditions or early GC at baseline than in those without.

\section{Clinical argument regarding GC risk following $\boldsymbol{H}$. pylori treatment}

As mentioned above, the GC risk after $H$. pylori eradication is different between primary GC that develops in healthy individuals or peptic ulcer patients and MGC. According to a survey conducted in Japan by Mori et al., ${ }^{72}$ the annual incidences of primary GC and MGC ranged from $0.1 \%$ to $0.5 \%$ and from $0.8 \%$ to $4.1 \%$, respectively. In their large-scale, multicenter cohort study, the annual incidence of MGC after $H$. pylori eradication was relatively high (3.0\%, cumulative incidence 15.0\% at 5 years after eradication), and was similar to the incidence found in another study from Japan. ${ }^{14}$ In addition, the incidence in the multicenter cohort study by Mori et al. (29.9 cases per 1,000 person-years $)^{72}$ was double the incidence found in other studies (14.1 cases per 1000 person-years ${ }^{11}$ and 14.7 cases per 1,000 person-years ${ }^{73}$ ). This incidence of MGC was also significantly higher than that found by Choi et al. (estimated incidence: approximately 5.7 to 7.5 cases per 1,000 person-years). ${ }^{15,71}$ This discrepancy may depend on the quality of the endoscopy and the timing of the surveillance following the eradication in addition to the population of high-risk patients enrolled in those studies, such as the proportion with risk factors including male sex, severe AG and IM. It was noteworthy that the ORs differed among countries in the subgroup analysis of a meta-analysis (OR, 0.39; 95\% CI, 0.31 to 0.49 in Japan and Korea vs OR, 0.62; 95\% CI, 0.42 to 0.91 in China and Colombia; $\mathrm{p}=0.04) .{ }^{10}$ In addition, subgroup analysis in this meta-analysis showed a difference in the endoscopic techniques used in the different countries and showed that higher incidences of MGC were found in areas with superior endoscopic detection of early lesions, thus suggesting that early identification of individuals with precan-

Table 1. Preventive Effects of Helicobacter pylori Eradication on Metachronous and Primary GC Development

\begin{tabular}{|c|c|c|c|c|}
\hline \multirow{2}{*}{ Author } & \multicolumn{2}{|c|}{ Metachronous GC } & \multicolumn{2}{|c|}{ Primary GC } \\
\hline & $\mathrm{IRR} / \mathrm{RR} / \mathrm{OR}$ & $95 \% \mathrm{CI}$ & $\mathrm{IRR} / \mathrm{RR} / \mathrm{OR}$ & 95\% CI \\
\hline Lee et $\mathrm{al}^{4}{ }^{4}$ & $0.46^{*}$ & $0.35-0.60$ & $0.62^{*}$ & $0.49-0.79$ \\
\hline Chen et $a l^{5}$ & $0.52^{\dagger}$ & $0.31-0.87$ & $0.70^{\dagger}$ & $0.49-0.99$ \\
\hline Yoon et al. ${ }^{6}$ & $0.42^{\ddagger}$ & $0.32-0.56$ & - & - \\
\hline Jung et al. ${ }^{7}$ & $0.392^{\ddagger}$ & $0.259-0.593$ & - & - \\
\hline Bang et al. ${ }^{8}$ & $0.467^{\dagger}$ & $0.362-0.602$ & - & - \\
\hline Xiao et al. ${ }^{9}$ & $0.50^{\dagger}$ & $0.41-0.61$ & - & - \\
\hline Sugano et al. ${ }^{10}$ & $0.51^{\ddagger}$ & $0.40-0.64$ & $0.41^{\ddagger}$ & $0.32-0.52$ \\
\hline
\end{tabular}

GC, gastric cancer; IRR, incidence rate ratio; RR, risk ratio/relative ratio; OR, odds ratio; CI, confidence interval.

${ }^{*} \mathrm{IRR} ;{ }^{\dagger} \mathrm{RR} ;{ }^{\ddagger} \mathrm{OR}$. 
cerous gastric conditions and GC at enrollment using endoscopy might play a significant role in improving the effect of eradication therapy. ${ }^{10}$

\section{CHEMOPREVENTIVE EFFECTS OF ASPIRIN ON MGC DE- VELOPMENT}

As described above, MGC occurs to some degree in patients in whom $H$. pylori had been eradicated following ER for early GC. Therefore, other combination treatments are needed to reduce the risk of GC after H. pylori eradication. To date, numerous reports have shown that aspirin and NSAIDs are associated with a reduced risk of GC.

\section{Review of RCTs}

Generally, it may be impossible to perform a randomized prevention trial to determine the preventive effects of aspirin on GC due to the cost, time, and risk of adverse events such as gastrointestinal bleeding. However, several RCTs have been reported. When looking at studies that evaluated 20 or more patients, no protective effect on GC was identified. ${ }^{74-76}$ Among these studies, one RCT showed that aspirin reduced GC death if the scheduled trial treatment was continued for 10 to 20 years (HR, 0.42; 95\% CI, 0.23 to 0.79). ${ }^{76}$

\section{Review of meta-analyses}

Most of the studies on regular aspirin use and GC have reported that this intervention is associated with a statistically significant reduction in GC risk; the RR ranged from 0.61 to $0.81{ }^{16,20,21,77,78}$ However, two meta-analyses reported that there was no statistically significant association between aspirin use and GC risk. ${ }^{79,80}$ A meta-analysis by Yang et al. ${ }^{80}$ showed that the effect of aspirin was seen in noncardiac GC (OR, 0.62; 95\% CI, 0.55 to 0.69). In a meta-analysis by Abnet et al., ${ }^{79}$ aspirin use was associated with a reduced risk of noncardiac GC (OR, 0.64; 95\% CI, 0.52 to 0.80), and nonaspirin NSAIDs had a significant association with both cardiac (OR, 0.80; 95\% CI, 0.67 to 0.95) and noncardiac (OR, $0.68 ; 95 \% \mathrm{CI}, 0.57$ to 0.81 ) GCs. Thus, the effect may be different between aspirin and nonaspirin NSAIDs.

It is noteworthy that when stratifying by study design, a statistically significantly reduced risk of GC was seen in cohort and RCT studies (OR, 0.72; 95\% CI, 0.62 to 0.84) but not in hospitalbased (OR, 0.71; 95\% CI, 0.49 to 1.02) or population-based (OR, $0.88 ; 95 \%$ CI, 0.51 to 1.54 ) studies. ${ }^{80}$ With regard to the causes of this discrepancy, Qiao et al. ${ }^{21}$ pointed out that the cases in the case-control studies might have a recall bias, and the studies may then have tended to overestimate the risk of cancer associated with aspirin use. Another possible explanation is that misclassification or measurement errors with regard to aspirin use in the cohort studies might have distorted the association, because most of the analyses were based on baseline data, and there might have been a discrepancy between initial recruitment and subsequent aspirin consumption. ${ }^{21}$

\section{Clinical argument regarding the chemopreventive effect of aspirin on GC risk}

The frequency, duration, and dose of the aspirin treatment may influence the results. A meta-analysis by Kong et al. ${ }^{19}$ showed that short- or medium-term ( $\leq 5$ years) use of aspirin or selective COX-2 inhibitors is probably a more effective approach to reduce GC risk. Meanwhile, other reports showed that the effect was most prominent with longer aspirin use, ${ }^{22}$ particularly aspirin use for more than 3 years, ${ }^{18} 4$ years, ${ }^{81}$ or 5 years. $^{21,78,82}$ There is also a report that showed that the duration of aspirin use was not associated with GC risk. ${ }^{20}$ In addition, the association between the frequency of aspirin use and the effect on GC risk remains controversial: high-frequency ( $>30$ times per month) intake of aspirin/NSAIDs had efficacy for GC prevention in one study ${ }^{19}$ but did not show a significant association in another. ${ }^{20}$ In a third study, low-frequency ( 1 to 4.5 times per week) intake of aspirin/NSAIDs was effective for preventing GC. ${ }^{81}$ A recent general population-based study from South Korea revealed that the effect was statistically significant when the cumulative dose was $>3$ defined daily dose-years. ${ }^{22}$

\section{Effects of aspirin from the perspective of $H$. pylori infec-} tion

Yang et $a .^{80}$ demonstrated in their meta-analysis that an effect of aspirin was seen in $H$. pylori-infected individuals (OR, $0.62 ; 95 \%$ CI, 0.42 to 0.90 ). Some studies also showed in stratified analysis that the chemopreventive effect of aspirin/NSAIDs was higher in $H$. pylori-infected subjects than in $H$. pylori-negative subjects. ${ }^{20,83-85}$ However, as $H$. pylori status was judged using only $H$. pylori IgG antibody in those previous studies, ${ }^{20,83-85}$ the diagnosis of $H$. pylori infection was not definitive. Furthermore, in those studies, the $H$. pylori-negative subjects included two subtypes who were completely different in terms of their GC risk: that is, $H$. pylori-uninfected patients and patients who were suspected of having naturally eradicated $H$. pylori. Cheung et al. ${ }^{82}$ recently reported that the protective effect of aspirin appeared to be larger in $H$. pylori-eradicated subjects (HR, 0.30 ; 95\% CI, 0.15 to 0.61). Therefore, further studies are needed to clarify the association.

\section{MOLECULAR ALTERATIONS IN THE PRECANCEROUS CONDITIONS OF THE GASTRIC MUCOSA}

In 2014, a landmark study by The Cancer Genome Atlas proposed four subtypes of GC: (1) Epstein-Barr virus-positive (8.8\%); (2) microsatellite unstable/instability (21.7\%); (3) genomically stable (19.7\%); and (4) chromosomally unstable/ chromosomal instability (CIN) (49.8\%). ${ }^{86}$ To date, many studies have reported that a lot of molecular events, including promoter hypermethylation of multiple tumor-related genes, are associ- 

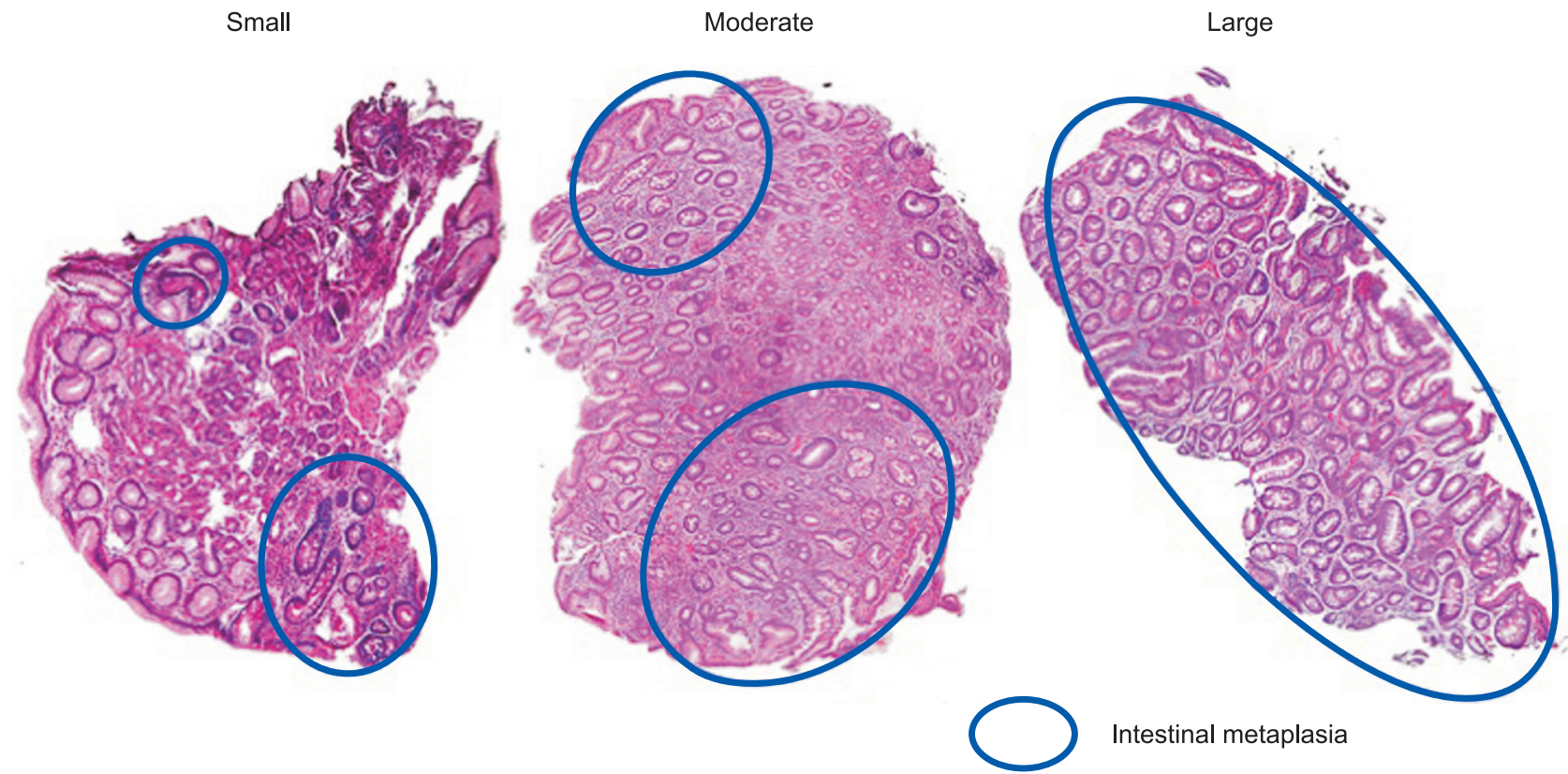

Intestinal metaplasia

Fig. 1. Biopsy specimens obtained from the gastric mucosa. Various numbers (small to large) of intestinal metaplastic glands are included in the samples (H\&E, ×200).

ated with GC and precancerous conditions of the stomach. The strong influence of methylation accumulation on GC risk is considered to be due to the major contribution to gastric carcinogenesis, along with mutations, of aberrant DNA methylation that is induced by $H$. pylori infection in gastric epithelial cells. ${ }^{34}$ It has been considered, however, that tumor-suppressor genes are more inactivated by DNA methylation than by mutations in gastric carcinogenesis, thus indicating that GC is an epigenetic disease. ${ }^{39}$ Recent review articles have summarized the triggers and the most-deregulated molecular pathways involved in $H$. pylori gastric carcinogenesis. ${ }^{35,87}$ Currently, the dysregulation of noncoding RNAs, e.g. microRNAs (miRNAs), such as the methylation of several tumor-suppressor miRNAs, is also considered to play important roles in the pathogenesis of GC. Several cancer risk biomarkers are also discussed in the above-mentioned studies. However, there have been few studies on the changes of DNA methylation after $H$. pylori eradication, especially in patients with IM, gastric dysplasia or GC.

\section{Effects of $\boldsymbol{H}$. pylori eradication on molecular events}

GC develops through the accumulation of molecular alterations from the precancerous conditions to GC. ${ }^{25-50}$ Over 10 years ago, it was reported that $H$. pylori eradication can significantly reduce gene methylation in the chronic gastritis mucosa, thereby delaying or reversing $H$. pylori-induced gastric carcinogenesis. ${ }^{26-28,30} \mathrm{Kim}^{88}$ reviewed the changes of molecular events in detail in a recent article. Chan et al. ${ }^{25}$ first showed that methylation of the E-cadherin $(\mathrm{CDH1})$ promoter was reversed after $H$. pylori eradication, and that the disappearance of E-cadherin methylation may be important for preventing the future development of GC. However, studies on the changes in the molecular phenotype in the background mucosa following $H$. pylori treatment in patients who have undergone ER for gastric neoplasms are limited..$^{31,32,38,41,45}$ Recently, the biomarkers for GC development after $H$. pylori eradication were discussed, and it was found that residual GC-related molecular events, even after $H$. pylori eradication, may be a surrogate marker of GC. . $^{30,32,34,38,41,45,46}$ The $\mathrm{CpG}$ island methylator phenotype (CIMP), which is characterized by extensive hypermethylation of multiple $\mathrm{CpG}$ islands within the genome, is currently recognized as one of the major mechanisms in gastric carcinogenesis, ${ }^{89}$ although there is no gold standard with respect to gene panels or the number of marker thresholds used to define CIMP. ${ }^{90}$ We recently reported that CIMP in IM might have potential as a biomarker of GC development. ${ }^{38}$ Additionally, it has been reported that the DNA methylation of several miRNA genes including miR-124a-3, miR-34b/c, and $m i R-137$ in the background mucosa was associated with an increased risk of developing primary $\mathrm{GC}^{47}$ and MGC. ${ }^{33,34,46,48,49}$ The highest quartile of the miR-124a-3 methylation level had a significant HR (3.0; 95\% CI, 1.58 to 5.72) in a 5-year follow-up of a multicenter prospective cohort study. ${ }^{34}$ Suzuki et al. ${ }^{49}$ reported that $m i R-34 b / c$ methylation, particularly in the corpus, showed the strongest association with the risk of MGC (HR, 9.00; 95\% CI, 1.56 to 52.01). However, in several of these studies, both $H$. pylori-negative (suspected to be naturally eradicated) and $H$. pylori-eradicated patients were treated as $H$. pylori-negative patients. $33,34,49$

Microsatellite instability (MSI) has been identified in about $20 \%$ of both IM and intraepithelial neoplasms, and is considered 


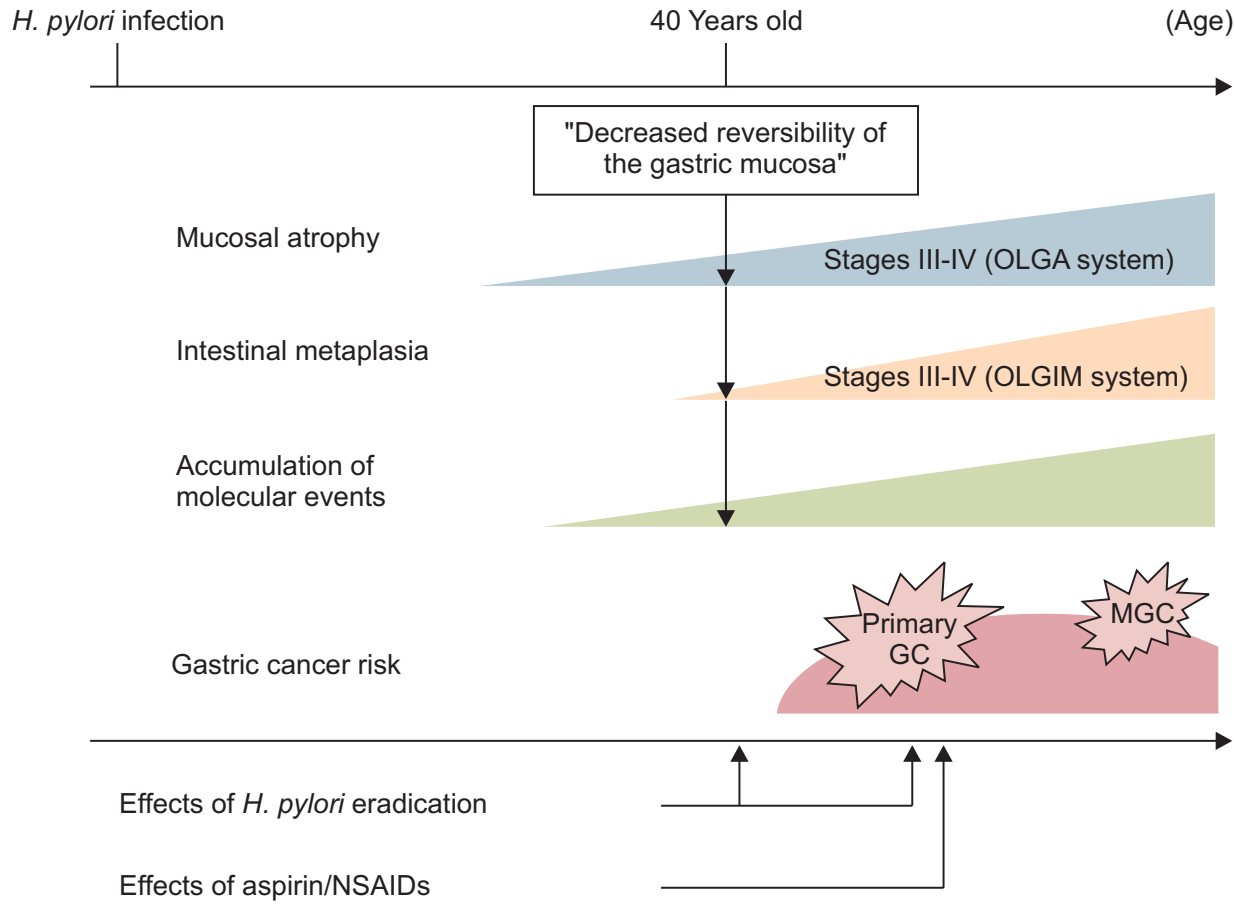

Fig. 2. Schematic of the association between gastric cancer (GC) risk and the effects of Helicobacter pylori eradication and aspirin/nonsteroidal anti-inflammatory drugs (NSAIDs) $H$. pylori eradication when the patient is in his or her 40s or prior to the "decreased reversibility of the gastric mucosa" may suppress GC development. With regard to the reduction in metachronous GC (MGC) risk, the treatment may be effective from a long-term perspective. Longterm aspirin/NSAID use may have additional effects in reducing MGC risk after $H$. pylori eradication. OLGA, operative link on gastric atrophy; OLGIM, operative link on gastric intestinal metaplasia. to be among the earliest molecular changes occurring in the oncogenic cascade. ${ }^{87}$ However, there have been few reports on the changes of MSI and CIN (loss of heterozygosity, LOH) after H. pylori therapy. Our own previous studies ${ }^{32,45}$ were exceptions, but in those reports we investigated MSI and LOH only in patients with IM and not in the context of atrophy. These molecular alterations in IM did not change significantly after the eradication, even in patients who underwent ER for early GC. Similarly, studies on the changes of somatic mutation following $H$. pylori eradication are rare. We analyzed the KRAS mutation in IM, and showed that (1) the prevalence of KRAS mutation in patients with IM without cancer was significantly higher than that in patients with intestinal-type GC; (2) KRAS mutations significantly declined in patients with chronic gastritis; (3) KRAS mutations in IM were related to lymphocyte infiltration caused by the bacteria; (4) AGT transformation in the mutation pattern was not affected by the treatment, and some mutations in KRAS may be selected by the eradication; and (5) H. pylori eradication before the development of stable mutations will likely halt the risk of $\mathrm{GC}^{50}$

\section{Effects of aspirin on molecular events}

There have been only a few studies on molecular alterations in the gastric mucosa in patients taking regular aspirin; ${ }^{37,91}$ to our knowledge, there have been no studies on the changes of somatic mutations and CIN by aspirin administration. Tahara et al. ${ }^{91}$ showed that chronic aspirin use was associated with a lower risk of $C D H 1$ methylation, especially in $H$. pylori-positive subjects (nonuser [49.5\%] vs user [19.0\%]: OR, 0.24; 95\% CI, 0.08 to $0.76 ; \mathrm{p}=0.01$ ). Our previous study also showed that long-term aspirin use ( $\geq 3$ years) was associated with a significant reduction of $C D H 1$ methylation in atrophy (OR, 0.15; 95\% CI, 0.06 to $0.41 ; p=0.0002$ ), but was less effective in reversing the methylation that occurred in IM. ${ }^{37}$ MSI did not change significantly after taking aspirin in either patient with atrophy or those with IM. $^{37}$ Interestingly, CIMP in IM, which is a surrogate marker of $\mathrm{GC}{ }^{38}$ decreased in patients with chronic gastritis who regularly took aspirin for more than three years. ${ }^{37}$ Therefore, long-term aspirin use, as an additional chemopreventive therapy, may help prevent GC development in patients with IM (Fig. 1).

\section{Problems of molecular analysis in precancerous condi- tions}

According to a meta-analysis by Chen et al., ${ }^{5}$ patients with IM or dysplasia could not benefit from the effect of $H$. pylori treatment on the risk of GC, thus indicating that the presence of IM seems to constitute a "point of no return" regardless of H. pylori eradication. ${ }^{67}$ Indeed, we reported that the incidence of molecular events related to carcinogenesis was significantly higher in IM than in atrophy (non-IM), irrespective of the presence or absence of $H$. pylori infection and GC in the background mucosa, ${ }^{37,38}$ and $H$. pylori eradication and long-term aspirin use were less effective in reversing the methylation that occurred in IM than in atrophy even in a long-term follow-up (median follow-up period: 5 years in $H$. pylori-eradication cases and 6.3 years in NSAIDs/aspirin users). ${ }^{37,38}$ Additionally, $H$. pylori eradication was associated with a significant reduction of CIMP, CDH1, and miR-124a-3 methylation only in atrophy but not in IM. $^{38}$ To date, we have analyzed molecular events in atrophy and IM separately, as precancerous conditions, using laser cap- 
ture microdissection (LCM). This procedure (i.e., LCM), might provide more information from atrophic or IM glands than would the use of whole tissue material with regard to gastric carcinogenesis in the precancerous conditions. ${ }^{32,37,38,92}$ Although it has been reported that $m i R-124 a-3$ and $m i R-34 b / c$ methylations in the background mucosa were associated with an increased risk of developing MGCs, ${ }^{33,34,48,49}$ in our previous study the methylations of miR-124a-3 and miR-34c were mostly observed in IM, and only rarely in atrophy. ${ }^{38}$ Thus, these results indicate that the methylation of these miRNA genes might be a specific marker expressed in IM and might not necessarily be a risk marker for $\mathrm{GC}^{38}$ In all previous studies except ours ${ }^{32,37,38}$ and a study by Huang et al., ${ }^{36}$ whole biopsy tissues were used for molecular analysis, and the differences in molecular alterations between atrophy and IM were not evaluated. The GC risk is generally considered to relate directly to the extent of IM. ${ }^{5,57}$ Therefore, the results in several of the studies ${ }^{33,34,48,49}$ may be affected by the amount of IM glands contained in the biopsy samples (Fig. 2). Shin et al. ${ }^{31}$ investigated the time course of DNA methylation after $H$. pylori treatment in a group of patients that included cases of gastric dysplasia and GC. They found that the eradication decreased the methylation levels in $L O X$, but not in $A P C$. Interestingly, the decrease in the methylation level in MOS after $H$. pylori eradication was significant among controls (cases without organic gastric or esophageal diseases) without IM; however, it was not observed among patients with IM or those with dysplasia or GC. Shin et al. speculated that the methylation level of MOS in patients with IM could be a surrogate marker for MGC risk. These results may support our results and indicate that $H$. pylori eradication decreases aberrant DNA methylation in a gene-specific manner.

\section{CONCLUSIONS}

Based on the clinical studies, $H$. pylori eradication and aspirin use are likely to prevent the development of MGC. Meta-analyses and RCTs show that $H$. pylori eradication reduces the risk of MGC development in patients who were followed-up for a long term after the eradication ( $\geq 5$ years). Interestingly, recent studies with long-term follow-up reported that AG and IM were reversed by $H$. pylori treatment. ${ }^{10,64}$ However, these clinical results diverged from the results of molecular pathological analyses. That is, molecular pathological analysis showed that IM rather than atrophy was closely associated with GC, thus indicating the concept of a "point of no return," ${ }^{, 67}$ in which the benefits of $H$. pylori eradication diminish after the development of IM in accompaniment with molecular changes. However, there have been few long-term follow-up studies on the changes of molecular events after $H$. pylori eradication and aspirin use. As Rugge et al. ${ }^{60}$ reported, $H$. pylori eradication in subjects with advanced stages (III-IV) according to the OLGA staging may not abolish the risk for neoplastic progression. In the future, there- fore, the discovery of precise biomarkers of MGC and the longterm follow-up of patients with those markers will be needed. $H$. pylori eradication alone may not reduce the MGC risk in the background mucosa in which atrophy and IM are extensively spread in the stomach. Thus, other combination treatments, that is, chemopreventive drugs such as aspirin or NSAIDs, are required to more greatly reduce the risk (Fig. 2). Ultimately, the most important point may be that regular endoscopic surveillance should be performed in patients who have undergone ER of GC.

\section{CONFLICTS OF INTEREST}

No potential conflict of interest relevant to this article was reported.

\section{ORCID}

Jiro Watari

Toshihiko Tomita

Katsuyuki Tozawa

Tadayuki Oshima

Hirokazu Fukui

Hiroto Miwa https://orcid.org/0000-0003-1112-1054

https://orcid.org/0000-0003-1283-9009

https://orcid.org/0000-0001-5048-550X

https://orcid.org/0000-0001-6949-932X

https://orcid.org/0000-0002-8642-5784

https://orcid.org/0000-0001-9844-642X

\section{REFERENCES}

1. Correa P. Helicobacter pylori and gastric carcinogenesis. Am J Surg Pathol 1995;19 Suppl 1:S37-S43.

2. Asaka M, Kato M, Sakamoto N. Roadmap to eliminate gastric cancer with Helicobacter pylori eradication and consecutive surveillance in Japan. J Gastroenterol 2014;49:1-8.

3. International Agency for Research on Cancer (IARC). Helicobacter pylori eradication as a strategy for preventing gastric cancer: IARC working group reports, vol 8 [Internet]. Lyon: IARC [cited 2016 Dec 6]. Available from: https://publications.iarc.fr/Book-AndReport-Series/Iarc-Working-Group-Reports/-Em-HelicobacterPylori-Em-Eradication-As-A-Strategy-For-Preventing-GastricCancer-2014.

4. Lee YC, Chiang TH, Chou CK, et al. Association between Helicobacter pylori eradication and gastric cancer incidence: a systematic review and meta-analysis. Gastroenterology 2016;150:11131124

5. Chen HN, Wang Z, Li X, Zhou ZG. Helicobacter pylori eradication cannot reduce the risk of gastric cancer in patients with intestinal metaplasia and dysplasia: evidence from a meta-analysis. Gastric Cancer 2016;19:166-175.

6. Yoon SB, Park JM, Lim CH, Cho YK, Choi MG. Effect of Helicobacter pylori eradication on metachronous gastric cancer after endoscopic resection of gastric tumors: a meta-analysis. Helicobacter 2014; 19:243-248.

7. Jung DH, Kim JH, Chung HS, et al. Helicobacter pylori eradica- 
tion on the prevention of metachronous lesions after endoscopic resection of gastric neoplasm: a meta-analysis. PLoS One 2015;10:e0124725.

8. Bang CS, Baik GH, Shin IS, et al. Helicobacter pylori eradication for prevention of metachronous recurrence after endoscopic resection of early gastric cancer. J Korean Med Sci 2015;30:749-756.

9. Xiao S, Li S, Zhou L, Jiang W, Liu J. Helicobacter pylori status and risks of metachronous recurrence after endoscopic resection of early gastric cancer: a systematic review and meta-analysis. J Gastroenterol 2019;54:226-237.

10. Sugano K. Effect of Helicobacter pylori eradication on the incidence of gastric cancer: a systematic review and meta-analysis. Gastric Cancer 2019;22:435-445.

11. Fukase K, Kato M, Kikuchi S, et al. Effect of eradication of Helicobacter pylori on incidence of metachronous gastric carcinoma after endoscopic resection of early gastric cancer: an open-label, randomised controlled trial. Lancet 2008;372:392-397.

12. Choi IJ, Kook MC, Kim YI, et al. Helicobacter pylori therapy for the prevention of metachronous gastric cancer. N Engl J Med 2018;378:1085-1095.

13. Maehata Y, Nakamura S, Fujisawa K, et al. Long-term effect of Helicobacter pylori eradication on the development of metachronous gastric cancer after endoscopic resection of early gastric cancer. Gastrointest Endosc 2012;75:39-46.

14. Kato M, Nishida T, Yamamoto K, et al. Scheduled endoscopic surveillance controls secondary cancer after curative endoscopic resection for early gastric cancer: a multicentre retrospective cohort study by Osaka University ESD study group. Gut 2013;62:14251432.

15. Choi J, Kim SG, Yoon H, et al. Eradication of Helicobacter pylori after endoscopic resection of gastric tumors does not reduce incidence of metachronous gastric carcinoma. Clin Gastroenterol Hepatol 2014;12:793-800.

16. Algra AM, Rothwell PM. Effects of regular aspirin on long-term cancer incidence and metastasis: a systematic comparison of evidence from observational studies versus randomised trials. Lancet Oncol 2012;13:518-527.

17. Cao Y, Nishihara R, Wu K, et al. Population-wide impact of long-term use of aspirin and the risk for cancer. JAMA Oncol 2016;2:762-769.

18. Kim YI, Kim SY, Kim JH, et al. Long-term low-dose aspirin use reduces gastric cancer incidence: a nationwide cohort study. Cancer Res Treat 2016;48:798-805.

19. Kong P, Wu R, Liu X, et al. The effects of anti-inflammatory drug treatment in gastric cancer prevention: an update of a metaanalysis. J Cancer 2016;7:2247-2257.

20. Huang XZ, Chen Y, Wu J, et al. Aspirin and non-steroidal anti-inflammatory drugs use reduce gastric cancer risk: a dose-response meta-analysis. Oncotarget 2017;8:4781-4795.

21. Qiao Y, Yang T, Gan Y, et al. Associations between aspirin use and the risk of cancers: a meta-analysis of observational studies. BMC Cancer 2018;18:288.
22. Kim MH, Chang J, Kim WJ, Banerjee S, Park SM. Cumulative dose threshold for the chemopreventive effect of aspirin against gastric cancer. Am J Gastroenterol 2018;113:845-854.

23. Ulrich CM, Bigler J, Potter JD. Non-steroidal anti-inflammatory drugs for cancer prevention: promise, perils and pharmacogenetics. Nat Rev Cancer 2006;6:130-140.

24. Sostres C, Lanas A. Gastrointestinal effects of aspirin. Nat Rev Gastroenterol Hepatol 2011;8:385-394.

25. Chan AO, Lam SK, Wong BC, et al. Promoter methylation of Ecadherin gene in gastric mucosa associated with Helicobacter pylori infection and in gastric cancer. Gut 2003;52:502-506.

26. Leung WK, Man EP, Yu J, et al. Effects of Helicobacter pylori eradication on methylation status of E-cadherin gene in noncancerous stomach. Clin Cancer Res 2006;12:3216-3221.

27. Chan AO, Peng JZ, Lam SK, et al. Eradication of Helicobacter pylori infection reverses E-cadherin promoter hypermethylation. Gut 2006;55:463-468.

28. Perri F, Cotugno R, Piepoli A, et al. Aberrant DNA methylation in non-neoplastic gastric mucosa of $\mathrm{H}$. pylori infected patients and effect of eradication. Am J Gastroenterol 2007;102:1361-1371.

29. Sepulveda AR, Yao Y, Yan W, et al. CpG methylation and reduced expression of 06-methylguanine DNA methyltransferase is associated with Helicobacter pylori infection. Gastroenterology 2010;138:1836-1844.

30. Nakajima T, Enomoto S, Yamashita S, et al. Persistence of a component of DNA methylation in gastric mucosae after Helicobacter pylori eradication. J Gastroenterol 2010;45:37-44.

31. Shin CM, Kim N, Lee HS, et al. Changes in aberrant DNA methylation after Helicobacter pylori eradication: a long-term follow-up study. Int J Cancer 2013;133:2034-2042.

32. Kawanaka M, Watari J, Kamiya N, et al. Effects of Helicobacter pylori eradication on the development of metachronous gastric cancer after endoscopic treatment: analysis of molecular alterations by a randomized controlled trial. Br J Cancer 2016;114:21 29.

33. Asada K, Nakajima T, Shimazu T, et al. Demonstration of the usefulness of epigenetic cancer risk prediction by a multicentre prospective cohort study. Gut 2015;64:388-396.

34. Maeda M, Nakajima T, Oda I, et al. High impact of methylation accumulation on metachronous gastric cancer: 5-year followup of a multicentre prospective cohort study. Gut 2017;66:17211723.

35. Liu X, Meltzer SJ. Gastric cancer in the era of precision medicine. Cell Mol Gastroenterol Hepatol 2017;3:348-358.

36. Huang KK, Ramnarayanan K, Zhu F, et al. Genomic and epigenomic profiling of high-risk intestinal metaplasia reveals molecular determinants of progression to gastric cancer. Cancer Cell 2018;33:137-150.

37. Michigami Y, Watari J, Ito C, et al. Effects of long-term aspirin use on molecular alterations in precancerous gastric mucosa in patients with and without gastric cancer. Sci Rep 2017;7:13384.

38. Michigami Y, Watari J, Ito C, et al. Long-term effects of H. pylori 
eradication on epigenetic alterations related to gastric carcinogenesis. Sci Rep 2018;8:14369.

39. Ushijima T, Hattori N. Molecular pathways: involvement of Helicobacter pylori-triggered inflammation in the formation of an epigenetic field defect, and its usefulness as cancer risk and exposure markers. Clin Cancer Res 2012;18:923-929.

40. Kang GH, Lee S, Kim JS, Jung HY. Profile of aberrant CpG island methylation along the multistep pathway of gastric carcinogenesis. Lab Invest 2003;83:635-641.

41. Maekita T, Nakazawa K, Mihara M, et al. High levels of aberrant DNA methylation in Helicobacter pylori-infected gastric mucosae and its possible association with gastric cancer risk. Clin Cancer Res 2006;12(3 Pt 1):989-995.

42. Ando T, Yoshida T, Enomoto S, et al. DNA methylation of microRNA genes in gastric mucosae of gastric cancer patients: its possible involvement in the formation of epigenetic field defect. Int J Cancer 2009;124:2367-2374.

43. Dong CX, Deng DJ, Pan KF, et al. Promoter methylation of p16 associated with Helicobacter pylori infection in precancerous gastric lesions: a population-based study. Int J Cancer 2009;124:434439.

44. Li WQ, Pan KF, Zhang Y, et al. RUNX3 methylation and expression associated with advanced precancerous gastric lesions in a Chinese population. Carcinogenesis 2011;32:406-410.

45. Watari J, Moriichi K, Tanabe H, et al. Biomarkers predicting development of metachronous gastric cancer after endoscopic resection: an analysis of molecular pathology of Helicobacter pylori eradication. Int J Cancer 2012;130:2349-2358.

46. Maeda M, Yamashita S, Shimazu T, et al. Novel epigenetic markers for gastric cancer risk stratification in individuals after Helicobacter pylori eradication. Gastric Cancer 2018;21:745-755.

47. Steponaitiene R, Kupcinskas J, Langner C, et al. Epigenetic silencing of miR-137 is a frequent event in gastric carcinogenesis. Mol Carcinog 2016;55:376-386.

48. Suzuki H, Yamamoto E, Nojima M, et al. Methylation-associated silencing of microRNA-34b/c in gastric cancer and its involvement in an epigenetic field defect. Carcinogenesis 2010;31:20662073.

49. Suzuki R, Yamamoto E, Nojima M, et al. Aberrant methylation of microRNA-34b/c is a predictive marker of metachronous gastric cancer risk. J Gastroenterol 2014;49:1135-1144.

50. Watari J, Tanaka A, Tanabe $\mathrm{H}$, et al. K-ras mutations and cell kinetics in Helicobacter pylori associated gastric intestinal metaplasia: a comparison before and after eradication in patients with chronic gastritis and gastric cancer. J Clin Pathol 2007;60:921926.

51. Sipponen P, Kekki M, Haapakoski J, Ihamäki T, Siurala M. Gastric cancer risk in chronic atrophic gastritis: statistical calculations of cross-sectional data. Int J Cancer 1985;35:173-177.

52. de Vries AC, Haringsma J, Kuipers EJ. The detection, surveillance and treatment of premalignant gastric lesions related to Helicobacter pylori infection. Helicobacter 2007;12:1-15.
53. Kim N, Park YS, Cho SI, et al. Prevalence and risk factors of atrophic gastritis and intestinal metaplasia in a Korean population without significant gastroduodenal disease. Helicobacter 2008;13:245-255.

54. Dinis-Ribeiro M, Areia M, de Vries AC, et al. Management of precancerous conditions and lesions in the stomach (MAPS): guideline from the European Society of Gastrointestinal Endoscopy (ESGE), European Helicobacter Study Group (EHSG), European Society of Pathology (ESP), and the Sociedade Portuguesa de Endoscopia Digestiva (SPED). Endoscopy 2012;44:74-94.

55. Uemura N, Okamoto S, Yamamoto S, et al. Helicobacter pylori infection and the development of gastric cancer. N Engl J Med 2001;345:784-789.

56. Rugge M, Genta RM; OLGA Group. Staging gastritis: an international proposal. Gastroenterology 2005;129:1807-1808.

57. Capelle LG, de Vries AC, Haringsma J, et al. The staging of gastritis with the OLGA system by using intestinal metaplasia as an accurate alternative for atrophic gastritis. Gastrointest Endosc 2010;71:1150-1158.

58. Yun CY, Kim N, Lee J, et al. Usefulness of OLGA and OLGIM system not only for intestinal type but also for diffuse type of gastric cancer, and no interaction among the gastric cancer risk factors. Helicobacter 2018;23:e12542.

59. Rugge M, Genta RM, Fassan M, et al. OLGA gastritis staging for the prediction of gastric cancer risk: a long-term follow-up study of 7436 patients. Am J Gastroenterol 2018;113:1621-1628.

60. Rugge M, Meggio A, Pravadelli C, et al. Gastritis staging in the endoscopic follow-up for the secondary prevention of gastric cancer: a 5-year prospective study of 1755 patients. Gut 2019;68:11-17.

61. Yue H, Shan L, Bin L. The significance of OLGA and OLGIM staging systems in the risk assessment of gastric cancer: a systematic review and meta-analysis. Gastric Cancer 2018;21:579-587.

62. Shichijo S, Hirata Y, Niikura R, et al. Histologic intestinal metaplasia and endoscopic atrophy are predictors of gastric cancer development after Helicobacter pylori eradication. Gastrointest Endosc 2016;84:618-624.

63. Kimura K, Takemoto T. An endoscopic recognition of the atrophic border and its significance in chronic gastritis. Endoscopy 1969;3:87-97.

64. Hwang YJ, Kim N, Lee HS, et al. Reversibility of atrophic gastritis and intestinal metaplasia after Helicobacter pylori eradication: a prospective study for up to 10 years. Aliment Pharmacol Ther 2018;47:380-390.

65. Leung WK, Wong IOL, Cheung KS, et al. Effects of Helicobacter pylori treatment on incidence of gastric cancer in older individuals. Gastroenterology 2018;155:67-75.

66. Bae SE, Choi KD, Choe J, et al. The effect of eradication of Helicobacter pylori on gastric cancer prevention in healthy asymptomatic populations. Helicobacter 2018;23:e12464.

67. Wong BC, Lam SK, Wong WM, et al. Helicobacter pylori eradication to prevent gastric cancer in a high-risk region of China: a randomized controlled trial. JAMA 2004;291:187-194. 
68. Asaka M, Kato M, Graham DY. Strategy for eliminating gastric cancer in Japan. Helicobacter 2010;15:486-490.

69. Watari J, Chen N, Amenta PS, et al. Helicobacter pylori associated chronic gastritis, clinical syndromes, precancerous lesions, and pathogenesis of gastric cancer development. World J Gastroenterol 2014;20:5461-5473.

70. Ito M, Takata S, Tatsugami M, et al. Clinical prevention of gastric cancer by Helicobacter pylori eradication therapy: a systematic review. J Gastroenterol 2009;44:365-371.

71. Choi JM, Kim SG, Choi J, et al. Effects of Helicobacter pylori eradication for metachronous gastric cancer prevention: a randomized controlled trial. Gastrointest Endosc 2018;88:475-485.

72. Mori G, Nakajima T, Asada K, et al. Incidence of and risk factors for metachronous gastric cancer after endoscopic resection and successful Helicobacter pylori eradication: results of a large-scale, multicenter cohort study in Japan. Gastric Cancer 2016;19:911918.

73. Bae SE, Jung HY, Kang J, et al. Effect of Helicobacter pylori eradication on metachronous recurrence after endoscopic resection of gastric neoplasm. Am J Gastroenterol 2014;109:60-67.

74. Cook NR, Lee IM, Gaziano JM, et al. Low-dose aspirin in the primary prevention of cancer: the Women's Health Study: a randomized controlled trial. JAMA 2005;294:47-55.

75. Flossmann E, Rothwell PM; British Doctors Aspirin Trial and the UK-TIA Aspirin Trial. Effect of aspirin on long-term risk of colorectal cancer: consistent evidence from randomised and observational studies. Lancet 2007;369:1603-1613.

76. Rothwell PM, Fowkes FG, Belch JF, Ogawa H, Warlow CP, Meade TW. Effect of daily aspirin on long-term risk of death due to cancer: analysis of individual patient data from randomised trials. Lancet 2011;377:31-41.

77. Tian W, Zhao Y, Liu S, Li X. Meta-analysis on the relationship between nonsteroidal anti-inflammatory drug use and gastric cancer. Eur J Cancer Prev 2010;19:288-298.

78. Bosetti C, Rosato V, Gallus S, Cuzick J, La Vecchia C. Aspirin and cancer risk: a quantitative review to 2011. Ann Oncol 2012;23:1403-1415.

79. Abnet CC, Freedman ND, Kamangar F, Leitzmann MF, Hollenbeck AR, Schatzkin A. Non-steroidal anti-inflammatory drugs and risk of gastric and oesophageal adenocarcinomas: results from a co- hort study and a meta-analysis. Br J Cancer 2009;100:551-557.

80. Yang P, Zhou Y, Chen B, et al. Aspirin use and the risk of gastric cancer: a meta-analysis. Dig Dis Sci 2010;55:1533-1539.

81. Ye X, Fu J, Yang Y, Gao Y, Liu L, Chen S. Frequency-risk and duration-risk relationships between aspirin use and gastric cancer: a systematic review and meta-analysis. PLoS One 2013;8:e71522.

82. Cheung KS, Chan EW, Wong AYS, et al. Aspirin and risk of gastric cancer after Helicobacter pylori eradication: a territory-wide study. J Natl Cancer Inst 2018;110:743-749.

83. Wu CY, Wu MS, Kuo KN, Wang CB, Chen YJ, Lin JT. Effective reduction of gastric cancer risk with regular use of nonsteroidal anti-inflammatory drugs in Helicobacter pylori-infected patients. J Clin Oncol 2010;28:2952-2957.

84. Zaridze D, Borisova E, Maximovitch D, Chkhikvadze V. Aspirin protects against gastric cancer: results of a case-control study from Moscow, Russia. Int J Cancer 1999;82:473-476.

85. Akre K, Ekström AM, Signorello LB, Hansson LE, Nyrén O. Aspirin and risk for gastric cancer: a population-based case-control study in Sweden. Br J Cancer 2001;84:965-968.

86. Cancer Genome Atlas Research Network. Comprehensive molecular characterization of gastric adenocarcinoma. Nature 2014;513:202-209.

87. Rugge M, Genta RM, Di Mario F, et al. Gastric cancer as preventable disease. Clin Gastroenterol Hepatol 2017;15:1833-1843.

88. Kim N. Chemoprevention of gastric cancer by Helicobacter pylori eradication and its underlying mechanism. J Gastroenterol Hepatol 2019;34:1287-1295.

89. Chen HY, Zhu BH, Zhang CH, et al. High CpG island methylator phenotype is associated with lymph node metastasis and prognosis in gastric cancer. Cancer Sci 2012;103:73-79.

90. Hughes LA, Melotte V, de Schrijver J, et al. The CpG island methylator phenotype: what's in a name? Cancer Res 2013;73:58585868.

91. Tahara T, Shibata T, Nakamura M, et al. Chronic aspirin use suppresses CDH1 methylation in human gastric mucosa. Dig Dis Sci 2010;55:54-59.

92. El-Serag HB, Nurgalieva ZZ, Mistretta TA, et al. Gene expression in Barrett's esophagus: laser capture versus whole tissue. Scand J Gastroenterol 2009;44:787-795. 